\title{
PREFERÊNCIA DE VÔO DE NOVE ESPÉCIES DA FAMÍLIA SCOLYTIDAE (INSECTA: COLEOPTERA) NA AMAZÔNIA CENTRAL ${ }^{1}$
}

\author{
Raimunda Liége Souza de ABREU ${ }^{2}$, Cláudio Ruy Vasconcelos da FONSECA ${ }^{3}$, José \\ Camilo HURTADO GUERRERO ${ }^{3}$ e Estevão Vicente Cavalcanti Monteiro de PAULA ${ }^{2}$
}

RESUMO - Neste trabalho foi avaliada a preferência por altura de vôo de nove espécies da família Scolytidae, capturadas com armadilhas Escolitídeo/Curitiba e Marques/Carrano/Abreu, instaladas a $1 ; 3 ; 5 ; 7,5$ e $10 \mathrm{~m}$ de altura na Reserva Florestal Adolpho Ducke, no $\mathrm{km} 22$ da rodovia AM-010 (Manaus, AM). Para isto foi feita uma análise de variância e as médias comparadas pelo teste de Tukey. $\mathrm{O}$ resultado das análises mostraram que, independente da armadilha utilizada, Xyleborus affinis Eichhoff, Xyleborus sp.1 e Monarthrum sp.1 apresentaram preferência de vôo por alturas inferiores a $3 \mathrm{~m}$; Cryptocarenus heveae (Hagedorn) mostrou preferência por alturas superiores a $5 \mathrm{~m}$; e Hypothenemus obscurus Fabricius acima de 7,5m; Xylosandrus compactus Eichhoff não apresentou preferência por qualquer altura de vôo. Cryptocarenus diadematus Eggers, Hypothenemus eruditus Westwood e Premnobius cavipennis Eichhoff apresentaram divergência quanto à tendência de vôo, em relação às armadilhas utilizadas.

Palavras-chave: Amazonas, Coleoptera, Scolytidae, altura de vôo

Flight Preference of Nine Species of Scolytidae (Insecta: Coleoptera) Collected in the Central Amazonian Region

ABSTRACT - In this work the flight preference of nine species of the Scolytidae family was evaluated. These insects were captured with Escolitideo/Curitiba and Marques/Carrano/Abreu traps, installed at heights of $1,3,5,7,5$, and $10 \mathrm{~m}$, in the Adolpho Ducke forest reserve, located at the $\mathrm{km} 22$ of AM-10 road (Manaus, AM). An analysis of variance was carried out and the means were separated by the Tukey test. The results showed that, in both traps, the Xyleborus affinis Eichhoff species, Xyleborus sp.1 and Monarthrum sp.1, had preference for heights below $3 \mathrm{~m}$; Cryptocarenus heveae (Hagedorn) for heights above $5 \mathrm{~m}$, and Hypothenemus obscurus Fabricius, above $7,5 \mathrm{~m}$. Xylosandrus compactus Eichhoff had no preference for flight heights. On the other hand, Hypothenemus eruditus Westwood, Cryptocarenus diadematus Eggers, and Premnobius cavipennis Eichhoff presented discrepancy concerning flight preference, considering the kinds of trap utilised in this experiment.

Key-words: Amazon Region, Coleoptera, Scolytidae, height flight

\section{INTRODUÇÃO}

Como na maioria dos insetos, a atividade de vôo é um dos aspectos mais importantes da biologia dos
Scolytidae, porque através do vôo eles saem à procura de um hospedeiro favorável para o seu estabelecimento e desenvolvimento. Nesta atividade, o padrão de preferência de altura de vôo

'Parte da dissertação apresentada pelo primeiro autor ao programa de Pós-graduação do INPA/ UA, como requisito para obtenção do título de Mestre em Ciências Biológicas, Ảrea de Concentração Entomologia.

${ }^{2}$ Coordenação de Pesquisas em Produtos Florestais, INPA, Av. André Araújo, 2936, Caixa Postal 478, CEP 69.011-970, Manaus, AM, Brasil.

${ }^{3}$ Coordenação de Pesquisas em Entomologia, INPA, Av. André Araújo, 2936, Caixa Postal 478, CEP 69.011-970, Manaus, AM, Brasil. 
varia com a espécie e parece estar relacionado ao ponto em que ocorre a infestação na planta pelas espécies dessa familia. Algumas espécies de besouros, capturadas próximo ao nível do solo, geralmente atacam a parte inferior do tronco e aquelas encontradas em todas as alturas atacam diferentes partes da planta (Hosking \& Knight, 1975; Turnbow \& Franklin, 1980).

Ainda neste aspecto, Chapman \& Kinghorn (1958) e Hosking \& Knight (1975), utilizando armadilhas de impacto, tipo "janela", iscadas com etanol, coletaram maior número de indivíduos de Trypodendron lineatum (Oliver) próximo ao nível do solo e de acordo com o primeiro autor, a concentração foi maior em áreas onde haviam troncos caídos. Roling \& Kearby (1975), com as mesmas armadilhas posicionadas a $0,3 \mathrm{~m}$ e $5,5 \mathrm{~m}$ de altura, capturaram maior número de Xyleborus ferrugineus (Fabricius) a menos de 1,80 metros do solo. Gagne \& Kearby (1978) também com armadilhas entre 0,75 e 3,75 capturaram, apenas, um indivíduo de Xyleborus celsus Eichhoff e muitos exemplares de Monarthrum fasciatum Say. Turnbow \& Franklin (1980), concluíram que a altura de vôo de Hypothenemus dissimilis (Zimmermann) varia de $6 \mathrm{~m}$ a $8 \mathrm{~m}$. Zelaya (1985), utilizando armadilhas tipo ESALQ/84, iscadas com etanol, mostrou em plantios de Pinus spp., em Agudos, Estado de São Paulo, que Xyleborus affinis Eichhoff e $X$. ferrugineus foram mais capturadas a $4 \mathrm{~m}$ do solo; e Xyleborus spinulosus
Blandford, Xyleborus retusus Eichhoff e Xyleborus brasiliensis Eggers entre $8 \mathrm{~m}$ e $10 \mathrm{~m}$. Na mesma localidade, Flechtmann (1988) com o mesmo tipo de armadilha, coletou nove espécies de Scolytidae entre $8 \mathrm{~m}$ e $10 \mathrm{~m}$ do solo; seis entre $4 \mathrm{~m}$ e $6 \mathrm{~m}$; uma a $6 \mathrm{~m}$, enquanto quatorze não mostraram preferência por qualquer altura.

Em várias comunidades florestais do Paraná, Carrano-Moreira (1985), utilizou as armadilhas Escolitídeo/Curitiba modificada e Marques/Carrano a alturas de $0,5 \mathrm{~m}$ a $1,30 \mathrm{~m}$ do solo. Em Araucaria angustifolia (Bert.) O. Ktze e na mata nativa, todas as espécies apresentaram variância mínima de individuos a $1,30 \mathrm{~m}$. Os maiores índices de diversidade de espécies de Scolytidae foram obtidos a $1,30 \mathrm{~m}$ nas áreas de Pinus elliottii Engelm., Pinus taeda L., Eualyptus dunnii Maiden e mata nativa e a $0,5 \mathrm{~m}$ em $A$. angustifolia.

Assim, este trabalho foi realizado com o propósito de estudar a preferência por altura de vôo de nove espécies de Scolytidae, visando fornecer subsídios para futuros estudos de correlação entre altura de vôo e zona de ataque às plantas.

\section{MATERIAL E MÉTODOS}

Os estudos foram feitos com as nove espécies mais frequentes da família Scolytidae, oriundas de levantamento efetuado na Reserva Florestal Adolpho Ducke (Reserva Ducke), localizada na rodovia AM 010, km 22 (Manaus-AM). A vegetação característica da área foi 
descrita por vários autores, entre eles, Lechthaler (1956), Aubréville (1961) e Magalhães \& Alencar (1979); o clima, de acordo com a classificação de Köppen, é do tipo Afi. As armadilhas utilizadas na captura destas espécies, bem como o método de coleta, foram descritos por Abreu et al. (1997).

A avaliação da preferência de vôo das espécies pelas diferentes alturas, foi feita através da análise de variância com os dados transformados em $\log _{\mathrm{e}} \mathrm{x}+1 \mathrm{e}$ o nível de significância foi $\mathrm{p}<0,05$. Em casos de diferença significativa, as médias foram comparadas pelo teste de Tukey (Zar, 1974).

\section{RESULTADOS E DISCUSSÃO}

De acordo com os resultados, a maioria das espécies de Scolytidae estudadas, demonstraram preferência por altura de vôo (Tab. 1). X. affinis, Xyleborus sp.1 e Monarthrum sp.1 voaram preferencialmente a alturas inferiores a $3 \mathrm{~m}$. Zelaya (1985) e Flechtmann (1988) mostraram também que $X$. affinis teve preferência marcante por altura mais próxima do solo, em torno de $4 \mathrm{~m}$ e segundo Zelaya (1985), esta espécie centraliza seu ataque na parte inferior das árvores.

As espécies que mostraram preferência por maiores alturas de vôo foram Cryptocarenus heveae, cuja tendência manifestou-se por niveis superiores a $5 \mathrm{~m}$ e Hypothenemus obscurus entre $7,5 \mathrm{~m}$ e $10 \mathrm{~m}$ com a armadilha Marques/Carrano/Abreu e $10 \mathrm{~m}$ com a Escolitídeo/Curitiba. A altura de vôo de $C$. heveae foi semelhante aos resultados apresentados por Flechtmann (1988) mas foi discordante para $H$. obscurus.

As espécies $C$. diadematus, $H$. eruditus e $P$. cavipennis apresentaram preferência por diferentes alturas com as duas armadilhas. $C$. diadematus, foi mais capturada a $3 \mathrm{~m}$ com a armadilha Escolitídeo/Curitiba, o que diverge da Marques/Carrano/Abreu, onde apresentou números semelhantes nas diversas alturas. $H$. eruditus foi mais coletada acima de $10 \mathrm{~m}$ com a armadilha Marques/Carrano/ Abreu, e para a Escolitídeo/Curitiba não foram observadas diferenças entre as diferentes alturas. Premnobius cavipennis Eichhoff foi mais capturada com a armadilha Escolitídeo/Curitiba, a alturas superiores a $3 \mathrm{~m}$, não tendo demonstrado preferência por altura de vôo com Marques/Carrano/Abreu. Flechtmann (1988) mostrou que $C$. diadematus tem preferência por voar entre 6 e $8 \mathrm{~m}, H$. eruditus por altura acima de $10 \mathrm{~m}$ e $P$. cavipennis não apresentou preferência por altura de vôo.

A única espécie que não demonstrou preferência de vôo por qualquer das alturas testadas foi Xylosandrus compactus Eichhoff.

Algumas espécies voaram preferencialmente mais próximas ao solo, ou seja a $1 \mathrm{~m}$, como $X$. affinis, outras a $10 \mathrm{~m}$, como H. obscurus (Tab. 1; Fig. 1). Esta preferência por altura de vôo pode estar relacionada à zona de ataque ao hospedeiro (Chapman \& Kinghorn, 1958; Hosking \& Knight, 1975; Turnbow \& Franklin, 1980; Zelaya, 1985). Como algumas espécies apresentaram preferência por 
altura superior a $7,5 \mathrm{~m}$, isto sugere que elas podem ser encontradas em alturas superiores a esta, e que experimentos dessa natureza devem ser conduzidos para testar esta hipótese.

As espécies que foram predominantes em alturas próximas ao solo, mas que foram coletadas, em menores números, a maiores alturas, sugere a hipótese de que sua presença em alturas superiores seja apenas um exercício de vôo à procura de novos hospedeiros (Turnbow \& Franklin, 1980).

O fato dos dois tipos de armadilhas capturarem a mesma forma nas mesmas alturas sugere que ambas podem ser utilizadas para monitorar as populações dessas espécies

\section{CONCLUSÃO}

-X. affinis, Xyleborus sp.1 e Monarthrum sp.1 têm preferência de vôo em alturas inferiores a $3 \mathrm{~m}$;

- C. heveae voa preferen- cialmente a alturas superiores a $5 \mathrm{~m}$; - H. obscurus em alturas superiores a $7,5 \mathrm{~m}$;

- X. compactus não apresentou preferência por altura de vôo.

- C. diadematus, H. eruditus e $P$. cavipennis apresentam resultados diferentes quanto à preferência por altura de vôo com as duas armadilha.

\section{Bibliografia citada}

Abreu, R.L.S., C.R.V. da Fonseca, E.N. Marques. 1997. Análise das principais espécies de Scolytidae coletadas em floresta primária no Estado do Amazonas. Anais da Sociedade Entomológica do Brasil 26(3):527-535.

Aubreville, A. 1961, Étude ecologique des principales formations vegetales du Brésil et contribuition a la connaissace des forests de L'Amazonie Bresiliene. Centre Technique Forestier Tropical, Nogent-SurMarne, France. 268p.

Carrano-Moreira, A.F. 1985. Análise faunistica de Scolytidae em comunidade florestais no Estado do Paraná. Dissertação de

Tabela 1. Estatísticas descritivas da atividade de vôo de nove espécies da família Scolytidae coletadas com as armadilhas Marques/Carrano/Abreu e Escolitídeo/Curitiba (ANOVA; n $=$ tamanho da $\operatorname{amostra}=40$ )

a) Cryptocarenus diadematus

\begin{tabular}{|c|c|c|c|c|c|c|c|c|c|c|c|}
\hline \multicolumn{6}{|c|}{ Marques/Carrano/Abreu $(p>0,05)$} & \multicolumn{6}{|c|}{ Escolitideo/Curitiba $(p<0,001)$} \\
\hline $\begin{array}{l}\text { Alt. } \\
\text { (m) }\end{array}$ & Média & D.P & E.P. & $\begin{array}{l}\mathrm{N}^{0} \text { de } \\
\text { indiv. }\end{array}$ & Tukey & $\begin{array}{l}\text { Alt. } \\
\text { (m) }\end{array}$ & Média & D.P. & E.P. & $\begin{array}{l}\mathrm{N}^{2} \text { de } \\
\text { indiv. }\end{array}$ & Tukey* \\
\hline 1 & 0,05 & 0,32 & 0,05 & 2 & a & 1 & 0,00 & 0,00 & 0,00 & 0 & a \\
\hline 3 & 0,15 & 0,53 & 0,08 & 6 & a & 3 & 0,23 & 0,48 & 0,08 & 9 & $a b$ \\
\hline 5 & 0,13 & 0,33 & 0.05 & 5 & a & 5 & 0,28 & 0,64 & 0,10 & 11 & $a b$ \\
\hline 7,5 & 0,35 & 0.92 & 0,15 & 14 & a & 7,5 & 0,55 & 0.81 & 0,13 & 22 & b \\
\hline 10 & 0,23 & 0,48 & 0,08 & 9 & a & 10 & 0,38 & 0,81 & 0,13 & 15 & b \\
\hline
\end{tabular}


Tabela 1. Continuação

b) Cryptocarenus heveae

\begin{tabular}{ccccccccccccc}
\hline \multicolumn{4}{c}{ Marques/Carrano/Abreu $(p<0,001)$} & \multicolumn{4}{c}{ Escolitideo/Curitiba $(p<0,001)$} \\
\hline $\begin{array}{c}\text { Alt. } \\
(\mathrm{m})\end{array}$ & Média & D.P & E.P. & $\begin{array}{c}\mathrm{N}^{0} \text { de } \\
\text { indiv. }\end{array}$ & Tukey & $\begin{array}{c}\text { Alt. } \\
(\mathrm{m})\end{array}$ & Média & D.P. & E.P. & $\begin{array}{c}\mathrm{N}^{0} \text { de } \\
\text { indiv. }\end{array}$ & Tukey* \\
\hline 1 & 0,33 & 0,80 & 0,13 & 13 & a & 1 & 0,15 & 0,48 & 0,08 & 6 & $\mathrm{a}$ \\
3 & 0,45 & 0,93 & 0,15 & 18 & ab & 3 & 0,95 & 1,93 & 0,30 & 38 & ab \\
5 & 1,88 & 2,96 & 0,46 & 75 & c & 5 & 3,18 & 6,29 & 0,99 & 127 & ab \\
7,5 & 3,28 & 4,38 & 0,69 & 131 & c & 7,5 & 1,40 & 2,38 & 0,38 & 56 & $b$ \\
10 & 1,58 & 2,43 & 0,38 & 63 & bc & 10 & 3,73 & 5,71 & 0,90 & 149 & $b$ \\
\hline
\end{tabular}

c) Hypothenemus eruditus

\begin{tabular}{ccccccccccccc}
\hline \multicolumn{1}{c}{ Marques/Carrano/Abreu $(p<0,001)$} & \multicolumn{1}{c}{ Escolitídeo/Curitiba $(p>0,05)$} \\
\hline $\begin{array}{c}\text { Alt. } \\
(\mathrm{m})\end{array}$ & Média & D.P & E.P. & $\begin{array}{c}\mathrm{N}^{2} \text { de } \\
\text { indiv. }\end{array}$ & Tukey & $\begin{array}{c}\text { Alt. } \\
(\mathrm{m})\end{array}$ & Média & D.P. & E.P. & $\begin{array}{c}N^{2} \text { de } \\
\text { indiv. }\end{array}$ & Tukey* \\
\hline 1 & 0,38 & 0,70 & 0,11 & 15 & a & 1 & 0,28 & 0,60 & 0,09 & 11 & a \\
3 & 0,20 & 0,41 & 0,06 & 8 & a & 3 & 0,40 & 0,59 & 0,09 & 16 & a \\
5 & 0,40 & 0,63 & 0,10 & 16 & a & 5 & 0,25 & 0,49 & 0,07 & 10 & a \\
7,5 & 0,38 & 0,58 & 0,09 & 15 & a & 7,5 & 0,55 & 0,81 & 0,13 & 22 & a \\
10 & 1,13 & 1,26 & 0,20 & 45 & & $b$ & 10 & 0,43 & 0,71 & 0,11 & 17 & a \\
\hline
\end{tabular}

D. P. Desvio padrăo

E. P. Erro padrāo

- Médias seguidas pela mesma letra não difere significativamente

d) Hypothenemus obscurus

\begin{tabular}{ccccccccccccc}
\hline \multicolumn{4}{c}{ Marques/Carrano/Abreu $(\mathrm{p}<0,001)$} & \multicolumn{1}{c}{ Escolitideo/Curitiba $(p<0,001)$} \\
\hline $\begin{array}{c}\text { Alt. } \\
(\mathrm{m})\end{array}$ & Média & D.P & E.P. & $\begin{array}{l}\mathrm{N}^{2} \text { de } \\
\text { indiv. }\end{array}$ & Tukey & $\begin{array}{c}\text { Alt. } \\
(\mathrm{m})\end{array}$ & Média & D.P. & E.P. $\begin{array}{c}N^{0} \text { de } \\
\text { indiv. }\end{array}$ & Tukey* \\
1 & 0,03 & 0,16 & 0,03 & 1 & a & 1 & 0,10 & 0,50 & 0,08 & 4 & a \\
3 & 0,08 & 0,26 & 0,04 & 3 & a & 3 & 0,23 & 0,48 & 0,08 & 9 & a \\
5 & 0,30 & 0,59 & 0,09 & 10 & a & 5 & 0,48 & 1,01 & 0,16 & 19 & a \\
7,5 & 0,83 & 1,39 & 0,22 & 33 & & $b$ & 7,5 & 0,53 & 1,01 & 0,16 & 21 & a \\
10 & 1,28 & 1,56 & 0,25 & 51 & & b & 10 & 1,13 & 0,94 & 0,15 & 45 & b \\
\hline
\end{tabular}




\section{Tabela 1. Continuação}

e) Monarthrum sp.1

\begin{tabular}{ccccccccccccc}
\hline \multicolumn{4}{c}{ Marques/Carrano/Abreu $(p<0,001)$} & \multicolumn{1}{c}{ Escolitídeo/Curitiba $(p<0,001)$} \\
\hline $\begin{array}{c}\text { Alt. } \\
(\mathrm{m})\end{array}$ & Média & D.P & E.P. & $\begin{array}{l}\mathrm{N}^{0} \text { de } \\
\text { indiv. }\end{array}$ & Tukey & $\begin{array}{c}\text { Alt. } \\
(\mathrm{m})\end{array}$ & Média & D.P. & E.P. & $\begin{array}{c}N^{0} \text { de } \\
\text { indiv. }\end{array}$ & Tukey* $^{*}$ \\
\hline 1 & 1,28 & 1,60 & 0,25 & 51 & c & 1 & 0,70 & 0,91 & 0,14 & 28 & b \\
3 & 0,63 & 0,81 & 0,13 & 25 & bc & 3 & 0,28 & 0,45 & 0,07 & 11 & a \\
5 & 0,35 & 0,74 & 0,12 & 14 & ab & 5 & 0,20 & 0,46 & 0,07 & 8 & a \\
7,5 & 0,23 & 0,42 & 0,06 & 9 & ab & 7,5 & 0,05 & 0,22 & 0,03 & 2 & a \\
10 & 0,03 & 0,16 & 0,03 & 1 & a & 10 & 0,30 & 0,65 & 0,10 & 12 & a \\
\hline
\end{tabular}

\section{f) Premnobius cavipennis}

\begin{tabular}{|c|c|c|c|c|c|c|c|c|c|c|c|}
\hline \multicolumn{6}{|c|}{ Marques/Carrano/Abreu $(\rho>0,05)$} & \multicolumn{6}{|c|}{ Escolitídeo/Curitiba $(p<0,001)$} \\
\hline $\begin{array}{l}\text { Alt. } \\
\text { (m) }\end{array}$ & Média & D.P & E.P. & $\begin{array}{l}\mathrm{N}^{2} \text { de } \\
\text { indiv. }\end{array}$ & Tukey & $\begin{array}{l}\text { Alt. } \\
\text { (m) }\end{array}$ & Média & D.P. & E.P. & $\begin{array}{l}\mathrm{N}^{2} \text { de } \\
\text { indiv. }\end{array}$ & Tukey* \\
\hline 1 & 1,03 & 1,53 & 0,24 & 41 & a & 1 & 0,55 & $0, .85$ & 0,13 & 22 & a \\
\hline 3 & 1,63 & 2,75 & 0,43 & 65 & a & 3 & 1,40 & 1,58 & 0,25 & 56 & $b$ \\
\hline 5 & 1,68 & 1,59 & 0,25 & 67 & a & 5 & 1,43 & 1,45 & 0,23 & 57 & $b$ \\
\hline 7,5 & 1,65 & 1,78 & 0,28 & 66 & a & 7,5 & 1,55 & 1,71 & 0,27 & 62 & $b$ \\
\hline 10 & 1,88 & 1,86 & 0,29 & 75 & a & 10 & 1,88 & 1,32 & 0,21 & 75 & $\mathrm{~b}$ \\
\hline
\end{tabular}

D. P. Desvio padrão

E. P. Erro padrăo

- Médias seguidas pela mesma letra não difere significativamente

g) Xyleborus affinis

\begin{tabular}{|c|c|c|c|c|c|c|c|c|c|c|c|c|}
\hline \multicolumn{7}{|c|}{ Marques/Carrano/Abreu $(p<0,001)$} & \multicolumn{6}{|c|}{ Escolitídeo/Curitiba $(p<0,001)$} \\
\hline $\begin{array}{l}\text { Alt. } \\
\text { (m) } \\
\end{array}$ & Média & D.P & E.P. & $\begin{array}{l}\mathrm{N}^{0} \text { de } \\
\text { indiv. }\end{array}$ & & ukey & $\begin{array}{l}\text { Alt. } \\
(\mathrm{m})\end{array}$ & Média & D.P. & E.P. & $\begin{array}{l}N^{e} \text { de } \\
\text { indiv. }\end{array}$ & Tukey* \\
\hline 1 & 22,40 & 12,50 & 1,98 & 50 & & c & 1 & 32,78 & 16,06 & 2,54 & 1311 & d \\
\hline 3 & 15,23 & 11,00 & 1,74 & 42 & & c & 3 & 14,03 & 13,40 & 2,12 & 561 & $c$ \\
\hline 5 & 6,20 & 5,68 & 0,89 & 24 & & b & 5 & 4,56 & 3,67 & 0,58 & 181 & b \\
\hline 7,5 & 4,18 & 4,71 & 0,74 & 19 & a & $b$ & 7,5 & 3,00 & 3,61 & 0,57 & 120 & a \\
\hline 10 & 2,35 & 3,10 & 0,49 & 15 & $\mathrm{a}$ & & 10 & 2,65 & 4,03 & 0,64 & 106 & a \\
\hline
\end{tabular}


Tabela 1. Continuação

h) Xylosandrus compactus

\begin{tabular}{|c|c|c|c|c|c|c|c|c|c|c|c|}
\hline \multicolumn{6}{|c|}{ Marques/Carrano/Abreu $(p>0,05)$} & \multicolumn{6}{|c|}{ Escolitideo/Curitiba $(p>0,05)$} \\
\hline $\begin{array}{l}\text { Alt. } \\
\text { (m) }\end{array}$ & Média & D.P & E.P. & $\begin{array}{l}\mathrm{N}^{e} \text { de } \\
\text { indiv. }\end{array}$ & Tukey & $\begin{array}{l}\text { Alt. } \\
\text { (m) }\end{array}$ & Média & D.P. & E.P. & $\begin{array}{l}\mathrm{N}^{0} \text { de } \\
\text { indiv. }\end{array}$ & Tukey* \\
\hline 1 & 0.75 & 1,01 & 0.16 & 30 & $a$ & 1 & 0,68 & 0,97 & 0,15 & 27 & $a$ \\
\hline 3 & 0,40 & 0,78 & 0,12 & 16 & a & 3 & 0,73 & 0,93 & 0,15 & 29 & a \\
\hline 5 & 0,33 & 0,57 & 0,09 & 33 & a & 5 & 0,58 & 0,90 & 0,14 & 23 & a \\
\hline 7,5 & 0,55 & 0,71 & 0,11 & 22 & a & 7,5 & 0,35 & 0,53 & 0,08 & 14 & a \\
\hline 10 & 0,43 & 0,75 & 0,12 & 17 & $a$ & 10 & 0,33 & 0,57 & 0,09 & 13 & a \\
\hline
\end{tabular}

i) Xyleborus sp.1

\begin{tabular}{|c|c|c|c|c|c|c|c|c|c|c|c|}
\hline \multicolumn{6}{|c|}{ Marques/Carrano/Abreu $(p<0,001)$} & \multicolumn{6}{|c|}{ Escolitídeo/Curitiba $(p<0,001)$} \\
\hline $\begin{array}{l}\text { Alt. } \\
\text { (m) }\end{array}$ & Média & D.P & E.P. & $\begin{array}{l}\mathrm{N}^{e} \text { de } \\
\text { indiv. }\end{array}$ & Tukey & $\begin{array}{l}\text { Alt. } \\
\text { (m) }\end{array}$ & Média & D.P. & E.P. & $\begin{array}{l}\mathrm{N}^{0} \text { de } \\
\text { indiv. }\end{array}$ & Tukey* \\
\hline 1 & 1,30 & 1,98 & 0,31 & 52 & b & 1 & 1,85 & 2,68 & 0,42 & 74 & c \\
\hline 3 & 1,40 & 2,24 & 0,35 & 56 & b & 3 & 1,50 & 2,98 & 0.47 & 60 & bc \\
\hline 5 & 0,38 & 0,87 & 0,14 & 15 & a & 5 & 0,40 & 0,95 & 0,15 & 16 & $a b$ \\
\hline 7,5 & 0,23 & 0,73 & 0,12 & 9 & a & 7,5 & 0,05 & 0,22 & 0,03 & 2 & a \\
\hline 10 & 0,10 & 0,38 & 0,06 & 4 & a & 10 & $0, .08$ & 0,26 & 0,04 & 3 & a \\
\hline
\end{tabular}

D. P. Desvio padrăo

E. P. Erro padrão

- Médias seguidas pela mesma letra não difere significativamente

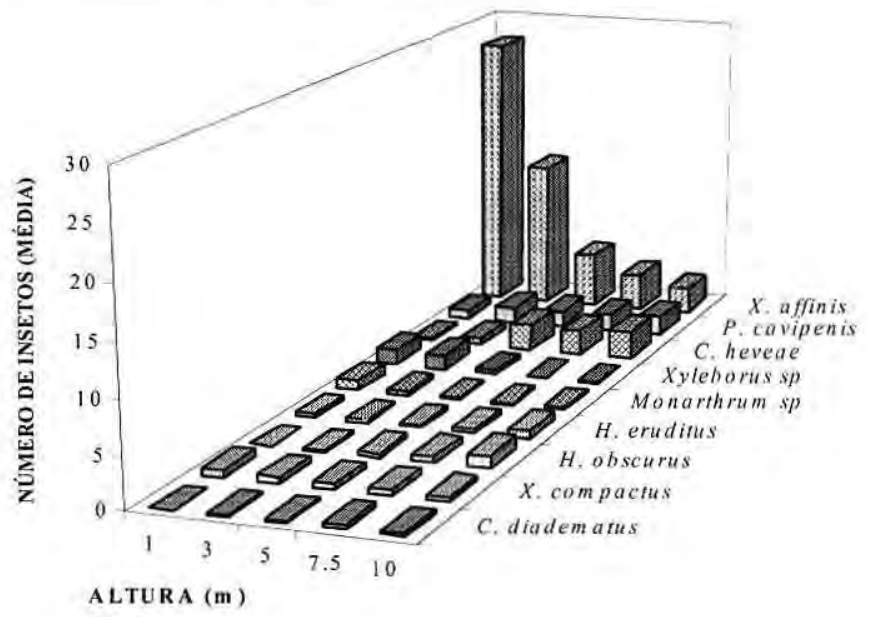

Figura 1. Distribuição de nove espécies da familia Scolytidae coletadas em floresta primária da Amazônia Central a alturas de 1;3;5;7,5 e 10m com as armadilhas Marques/Carrano/Abreu e Escolitídeo/Curitiba no período de março a outubro de 1993. 
mestrado, Universidade Federal Rural de Pernambuco, Recife, Pernambuco. 90p.

Chapman, J.A. 1958. Studies of flight and attack activity of the ambrosia beetle, Trypodendron lineatum (Oliver) and other scolytids. The Canadian Entomologist, 90(6):362-372.

Flechtmann, C.A.H. 1988. Altura de voo de Scolytidae em reflorestamento com pinheiros tropicais na regiào de Agudos, Estado de São Paulo. Dissertação de mestrado. Universidade de São Paulo/ Escola Superior de Agricultura Luiz de Queiroz, Piracicaba, São Paulo.132p.

Gagne, J.A.; W.H. Kearby, 1978. Host selection by Xyleborus celsus (Coleoptera: Scolytidae) in Missouri. The Canadian Entomologist, 110(10):1009-1013.

Hosking, G.P.; F.B. Knight, 1975. Flight habits of some Scolytidae in the spruce-fir type of Northern Maine. Annals of the Entomological Society of America, 68(5):917-921.

Lechthaler, R. 1956. Inventário das árvores de um hectare de terra firme da zona "Reserva Florestal Ducke", Municipio de Manaus. Série Botânica 3. Instituto Nacional de Pesquisas da Amazônia, Manaus. 7p

Magalhães, L.M.; J.C. Alencar, 1979. Fenologia do pau-rosa (Aniba duckei Kostermans) Lauraceae, em floresta primária da Amazônia Central, Acta Amazonica, 9:27-237.

Roling, M.P.; W.H. Kearby, 1975. Seasonal flight and vertical distribution of Scolytidae attracted to ethanol in a OakHickory forest in Missouri. The Canadian Entomologist, 107(12):315-1320.

Turnbow, Jr., R.H.; R.T. Franklin, 1980. Flight activity by Scolytidae in the Northeast Georgia Piedmont (Coleoptera). Journal of the Georgia Entomological Society, 15(1):26-37.

Zar, J H. 1974. Biostatistical analysis. PrenticeHall, Inc., Englewood Cliffs, New Jersey. $619 \mathrm{p}$.

Zelaya, M.R.M. 1985. Observações sobre o comportamento de Xyleborus spp. (Coleoptera: Scolytidae) em florestas de Pinus spp. na região de Agudos, Estado de São Paulo. Dissertação de mestrado. Universidade de São Paulo/Escola Superior de Agricultura Luiz de Queiroz, Piracicaba, São Paulo.88p. 